\title{
Perfil del hospital universitario del siglo XXI
}

\author{
Felicidad Rodríguez Sánchez
}

En una sociedad marcada por el avance continuo del conocimiento, al que no es ajeno el ámbito sanitario, todo hospital, en el nivel referencial que le corresponda, debe tener un cierto componente docente e investigador que incida sobre la calidad asistencial que presta. Pero, aun cuando todos los centros asistenciales del sistema sanitario contemplen ese doble aspecto, y todos ellos estén disponibles para desarrollar actividades formativas, la función primordial de la mayoría de los centros del sistema sanitario está focalizada esencialmente hacia la asistencia. Por ello, ante los retos actuales, cobra importancia la revalorización de centros asistenciales de excelencia, capaces de liderar la respuesta a los desafíos sanitarios del siglo XxI, especializados en formación e investigación, funciones que definen, precisamente, al hospital con denominación de universitario.

La formación del médico debe obedecer a un continuum, desde la enseñanza de grado/máster (primer y segundo ciclo del Espacio Europeo de Educación Superior) hasta la formación especializada, incluyendo la formación continuada y de otros posgrados, $y$, especialmente, la formación en investigación avanzada, el doctorado o tercer ciclo universitario. Durante los últimos años asistimos a la adaptación de los estudios de medicina a las pautas de la Declaración de Bolonia, una adaptación que ha tenido como eje la continuidad de la formación médica. Los nuevos planes de estudios han supuesto un avance en la adaptación a las nuevas exigencias de formación, con un gran trabajo colaborativo entre las facultades de medicina españolas y un gran trabajo en red con otras facultades europeas, como la Red Temática Europea Medine, para avanzar en la armonización de contenidos, competencias y estructuras, o para impulsar la movilidad y la investigación. Los nuevos planes de estudio ponen de relieve la necesidad de abordar aquellos aspectos que marcarán la práctica asistencial de los próximos años, al tiempo que han introducido cuestiones relevantes como ética, comunicación, gestión o investigación. Su aplicación ha supuesto la inmersión temprana del alumno en clínica, prácticamente desde segundo curso, así como la realización de un rotatorio final de sexto que plantea la filosofía de un residente 0 , avanzándose así hacia ese continuum en la formación, en la que el hospital representa un entorno clave. En los nuevos currículos cobra especial importancia la formación en investigación para alcanzar, de acuerdo con el Marco Europeo de Cualificaciones, el nivel académico de máster, que enlaza con el siguiente nivel, el de doctorado, para cuyo eficaz desarrollo es necesaria la colaboración estratégica entre el sistema universitario y el sanitario. El que la formación del médico responda, efectiva y eficazmente, a las necesidades planteadas exige un esfuerzo de colaboración entre la universidad y el sistema sanitario, un modelo que favorezca el continuum formativo y, en este contexto, el hospital universitario debería tomar especial relieve como espacio de encuentro y colaboración máxima entre las dos instituciones que tienen las responsabilidades asistenciales y académicas de las que dependen la formación de los futuros profesionales de la asistencia, de la investigación y de la docencia médica.

Sin embargo, los nuevos planes han puesto de manifiesto diversos problemas que se venían arrastrando de años atrás y que tienen que ver con dificultades en el desarrollo de la formación práctica de los estudiantes de medicina. A diferencia de lo que ocurre en la formación especializada, cuyos objetivos y estructura están bien regulados en el ámbito sanitario, en el caso de la formación de grado/máster esos objetivos quedan en la práctica mucho más desdibujados, con dificultades para la incorporación activa del estudiante en el proceso de enseñanza-aprendizaje en el entorno asistencial. No es el único problema. Observamos otras dificul-
Exdecana de la Facultad de Medicina de la Universidad de Cádiz. Diputada nacional

E-mail: felicidad.rodriguez@uca.es (c) 2013 FEM 
tades, como las que afectan a la adecuación entre el número de estudiantes y la capacidad formativa de los centros asistenciales, hasta las referentes a planificación estratégica de recursos humanos o a la coordinación entre las actividades asistenciales, docentes e investigadoras de los profesionales sanitarios. Para abordar estas cuestiones, conviene recordar el contexto en el que se desarrolla la formación médica en nuestro país, y que tiene que ver con:

- Los ámbitos competenciales sobre ese continuum de la formación médica.

- El marco actual en el que se desenvuelven los hospitales denominados universitarios.

Mientras que lo que ha venido en llamarse formación pregraduada (formación de grado o de grado/ máster según su nivel en el Marco Europeo de Cualificaciones) tiene carácter universitario, la formación especializada depende únicamente del sistema sanitario. Mientras que la formación especializada está claramente organizada en ese sistema, la formación de grado/máster debe obedecer al resultado de la colaboración entre dos instancias bien diferenciadas: la universidad y la institución sanitaria. Sin embargo, la formación de los estudiantes de medicina en el sistema sanitario adolece, con demasiada frecuencia, de una organización y unas pautas bien definidas que respondan a las necesidades formativas $y$, a este respecto, hay que tener en cuenta el marco normativo actual. Tras la incorporación de los antiguos hospitales clínicos al sistema sanitario, la Ley General de Sanidad de 1986 estableció las líneas generales de relación entre universidades e instituciones sanitarias. Establecía la necesidad de regular la dedicación a la investigación del personal docente, las bases de desarrollo de la carrera docente, investigadora y asistencial, así como las pautas generales para una mayor interrelación entre el sistema sanitario y el universitario, contemplando la participación de las universidades en los órganos de gobierno de los centros asistenciales y la de los representantes sanitarios en las juntas de facultad y de departamentos. El Real Decreto 1558/1986, en desarrollo de la Ley Orgánica de Reforma Universitaria de 1983 y de la Ley General de Sanidad, estableció las bases generales del régimen de conciertos entre las universidades y las instituciones sanitarias, con el objetivo de garantizar esos objetivos, y creó la figura del profesor universitario con plaza vinculada, que debía responder a las actividades del cuerpo docente de las áreas de salud, la docente, la investigadora y la asistencial, y, asimismo, se recogían particularidades para el acceso a la carrera universitaria de facultativos espe- cialistas. A raíz de ello, la colaboración entre las universidades y las instituciones sanitarias se plasmó en diversos convenios, que, con modalidades diferentes, han conducido a resultados diversos. Tras el paso de los años, existen muchas cuestiones de las señaladas que no han sido desarrolladas, o han sido insuficientemente abordadas, en los convenios de colaboración (sujetos, a su vez, a diferentes normas de carácter autonómico), lo que, sin duda, ha conducido a disfunciones con trascendencia efectiva en los aspectos docentes e investigadores. En ello influye la dificultad de integrar, de manera adecuada y eficiente, la cultura académica con la estrictamente asistencial. Algunos ejemplos son:

- Dificultades para la adecuada correspondencia y reconocimiento entre las funciones y responsabilidades docentes, investigadoras y asistenciales del profesorado vinculado, y para el desarrollo armónico de una carrera asistencial y académica entre el personal estatutario.

- Dificultades para compatibilizar la formación especializada con la formación en docencia e investigación de los que, necesariamente, serán los futuros profesores universitarios clínicos.

- Dificultades para que la docencia y la investigación ocupen un papel protagonista en los centros asistenciales. Con mucha frecuencia, se constata una ausencia de objetivos claros en docencia (especialmente de grado) que no suele ocupar un papel relevante en la gestión del centro, como tampoco se contempla adecuadamente en su estructura, en sus espacios, en su organización o en sus resultados.

- Dificultades para la eficaz participación mutua, universidad e institución sanitaria, en los órganos de dirección respectivos.

En el caso de muchos conciertos, el número de profesores con plaza vinculada se ha reducido de manera considerable durante el tiempo transcurrido, a la vez que se ha incrementado el número de alumnos por encima de la capacidad docente de los centros asistenciales, mientras que no se ha abordado en profundidad la regulación de la dedicación a la investigación del personal docente, el desarrollo de la carrera docente, investigadora y asistencial, ni las figuras de coordinación y representación. Obviamente, no basta el haber concertado un hospital en su totalidad ni disponer de una infraestructura suficiente para que un hospital, por obtener el calificativo de universitario, se convierta realmente en un hospital universitario. Aun cuando toda la estructura sanitaria esté disponible para la docencia, los hospitales universitarios, por sus propias carac- 
terísticas como punto de encuentro de los objetivos estratégicos del sistema universitario y del sanitario, deben redefinirse considerando los nuevos retos. Para ello es imprescindible poner en valor la especialización docente, investigadora e innovadora del hospital universitario, lo que debe traducirse en una excelencia en lo asistencial y en un motor de empuje y referencia para el conjunto del sistema sanitario; una especialización que debe impregnar toda la estructura del hospital y que implique una vocación de excelencia formativa e investigadora que debe iniciarse con la formación de calidad del médico de base y extenderse a la formación posgraduada y de doctorado, a la formación especializada y a la formación continuada. Además, la incorporación de España al Espacio Europeo de Educación Superior y al Espacio Europeo de Investigación marca nuevos retos a ambas instituciones, en términos de competitividad docente e investigadora, y por consiguiente asistencial, tanto a nivel nacional como internacional. Para los estudios de grado/máster existen nuevos requerimientos que persiguen una mejora de la calidad docente e investigadora, tomando relieve la garantía de la calidad de los nuevos títulos y de los contextos en los que éstos se desarrollan, con especial hincapié en la calidad de los recursos humanos y materiales dedicados a las actividades docentes e investigadoras. La acreditación del título está sujeta al cumplimiento de sus directrices, a los recursos materiales en los contextos en los que se adquieren los conocimientos y competencias pertinentes, y a la disponibilidad de recursos humanos necesarios en el número y en los niveles de acreditación necesarios. Ese contexto es, fundamentalmente, el hospitalario, por lo que se muestra imprescindible el esfuerzo conjunto de las instituciones universitaria y sanitaria para lograr esos requisitos, y ello pasa por el abordaje de cómo se desarrolla la formación médica en el entorno hospitalario y, más concretamente, por la actualización del perfil del hospital universitario. El hospital universitario del siglo XXI debe ser un centro de la máxima calidad asistencial, en el que las funciones académicas, docente e investigadora, sean claramente definitorias y con trascendencia real en su gestión, estructura, organización y funcionamiento. La investigación e innovación biomédica, la transferencia de conocimiento y la formación, en todos sus niveles, deben ser objetivos estratégicos para avanzar en una asistencia de la mayor excelencia, de manera que las tres funciones se desarrollen armónicamente con los mayores niveles de calidad. Para ello es imprescindible una puesta en valor del hospital universi- tario, así como afrontar y superar las dificultades identificadas.

\section{En términos organizativos}

El hospital universitario debe tener una estructura que equilibre su triple función, incorporando la cultura académica como algo propio. El carácter universitario precisa contemplar tanto la adquisición del conocimiento (investigación), como su transmisión (docencia) y su aplicación (asistencia), con una correcta adecuación de las estructuras asistenciales y académicas, un mayor nivel de interrelación entre el sistema universitario y el asistencial, así como una mayor conciencia de la disponibilidad de recursos comunes, lo que es especialmente relevante en el caso de los recursos humanos. Se necesita abordar la representación mutua en los órganos de dirección, pero, también, el desarrollo de fórmulas de coordinación más eficaces para el equilibrio armónico de la asistencia, la docencia y la investigación, lo que debe suponer un mayor relieve de la responsabilidad conjunta, y procedimientos que superen las limitaciones actuales y complementen la dinámica de comisiones mixtas cuya mecánica de funcionamiento, en muchos casos, no responde a las necesidades diarias. Para ello es necesario analizar la efectiva aplicación, y la correspondiente actualización, de las disposiciones que regulan las relaciones universidad-sistema sanitario.

\section{Las misiones docentes e investigadoras}

Deben ser características del hospital universitario, por lo que toda la estructura hospitalaria, sin excepción, debe estar realmente asociada a esas misiones. La docencia se debe plantear en todos los niveles, con especial atención a la colaboración entre el sistema universitario y el sanitario en la investigación, en el desarrollo de posgrados específicos y en el impulso del doctorado, incluyendo nuevos escenarios de docencia que aúnen los intereses asistenciales e investigadores para propiciar la mayor y mejor formación investigadora de los profesionales sanitarios. Ello incluye la disposición de espacios de investigación y educativos adecuados, con los recursos didácticos necesarios, así como el establecimiento de planes de docencia e investigación en las distintas unidades asistenciales, con la inclusión de objetivos en los contratos-programa, así como la garantía de que el alumno tenga un papel claro en el 
ámbito hospitalario. Todo ello acompañado de mecanismos de garantía de calidad, con los correspondientes indicadores y procedimientos de evaluación, que deben extenderse a la investigación con indicadores propios de la consideración de hospital universitario: tesis doctorales, proyectos de investigación, publicaciones, factor de impacto, etc., cuestiones todas ellas que deben plasmarse en la valorización de los resultados, con los consecuentes incentivos, y que deberían contemplar actuaciones conjuntas entre la universidad y la institución sanitaria en la identificación de fortalezas, la puesta en común de recursos y el desarrollo de planes estratégicos.

\section{Los recursos humanos}

Merecen especial atención, ya que un hospital universitario debe garantizar que todo su personal adquiere un compromiso con la docencia en los correspondientes niveles de responsabilidad, compromiso extendido a la investigación, lo que implica compatibilizar la triple función con el adecuado reconocimiento en el sistema sanitario y en el universitario, estimulando la carrera universitaria de los profesionales sanitarios. Esto incluye la formación en docencia e investigación de los residentes, mediante planes colaborativos entre la institución sanitaria y los departamentos universitarios, ya que los 'mires' representan la cantera de profesores e investigadores del futuro. Por otro lado, la docencia e investigación de calidad hace imprescindible la presencia de profesores del máximo nivel, con una más clara definición de la doble dependencia del profesorado vinculado, de sus derechos y deberes, y por el equilibrio entre su triple función con un esquema de dedicación que redunde en la excelencia de ambas instituciones. Es necesario disponer de un mapa básico de plazas vinculadas de los cuerpos docentes, que asegure la especialidad docente e investigadora, así como una estrategia de atracción y desa- rrollo de estas plazas en aras de la excelencia académica y asistencial: estrategia de incorporación a la actividad académica del personal asistencial; formación en docencia e investigación de los residentes; desarrollo de nuevas fórmulas de vinculación de profesionales; e incentivo del efectivo desarrollo de una carrera docente-asistencial-investigadora reconocida por ambas instituciones.

No son los anteriores los únicos aspectos que deben abordarse en la redefinición del hospital universitario. Un aspecto importante es, entre otros, el planteamiento de nuevos modelos de financiación de unos centros que deben ser referentes en asistencia, docencia e investigación ante los retos en salud del nuevo siglo. Y, para ello, resulta crucial que todas las instancias implicadas, políticas, universitarias y sanitarias, sean conscientes del valor estratégico de la docencia y la investigación para los retos en salud del siglo xxI, y del papel que los hospitales universitarios pueden representar para afrontar esos retos.

Bibliografía

1. Comision Europa. El marco europeo de cualificaciones para el aprendizaje permanente (EQF-MEC). Luxemburgo: Oficina de Publicaciones Oficiales de las Comunidades Europeas; 2009.

2. Directiva 2005/36/CE, del Parlamento Europeo y del Consejo, de 7 de septiembre de 2005, relativa al reconocimiento de cualificaciones profesionales. Diario Oficial de la Unión Europea L 255 de 30 de septiembre de 2005. p. 38.

3. Ley $14 / 1986$, de 25 de abril, General de Sanidad. Boletín Oficial del Estado n. ${ }^{\circ}$ 102, de 29 de abril de 1986. p. 15207-24.

4. Ley $44 / 2003$, de 21 de noviembre, de ordenación de las profesiones sanitarias. Boletín Oficial del Estado n. ${ }^{\circ} 280$, de 22 de noviembre de 2003. p. 41442-58.

5. Medine 2 Thematic Network in Medical Education. European Commision. Education and Culture DG. http://medine2.com.

6. Orden ECI/332/2008, de 13 de febrero, por la que se establecen los requisitos para la verificación de los títulos universitarios oficiales que habiliten para el ejercicio de la profesión de médico. Boletín Oficial del Estado n. ${ }^{\circ}$ 40, de 15 de febrero de 2008. p. 8351-5.

7. Real Decreto $1558 / 1986$, de 28 de junio, por el que se establecen las bases generales del régimen de conciertos entre las universidades y las instituciones sanitarias. Boletín Oficial del Estado n. ${ }^{\circ}$ 182, de 31 de julio de 1986. p. 27235-9. 


\section{El hospital como centro de enseñanza de la medicina: el alumno en el hospital}

Felipe Rodríguez de Castro

En los años setenta, estudiar medicina suponía, básicamente, realizar unos estudios teóricos. La mayor parte de los alumnos podía terminar su carrera sin haber visto un enfermo ingresado y los hospitales clínicos no servían tanto para realizar una enseñanza práctica directa, como para, en el mejor de los supuestos, extraer casos concretos y mostrarlos en la facultad. Para la mayor parte de los profesores, la actividad práctica por excelencia era la presentación de problemas clínicos, a veces con la presencia del paciente en el aula para dar al caso un sentido práctico y concreto. El estudiante de medicina no tenía la sensación de estar estudiando en un hospital, sino en una facultad, incluso aunque ésta estuviera adosada o incluida en el hospital universitario en cuestión. Esta situación era producto, por una parte, de una programación ineficaz y desequilibrada de los conocimientos a impartir y de las habilidades técnicas a adquirir por los estudiantes de medicina, $y$, por otra, del elevado número de alumnos y de la escasa dotación de profesorado.

\section{Hospital universitario: un repaso a la historia reciente}

En España no ha habido una auténtica tradición de hospital universitario. En las llamadas 'facultades clásicas', existían unos hospitales clínicos que eran propiedad de la universidad y que estaban vinculados a la facultad de medicina. Los profesores-médicos eran empleados de la universidad y participaban activamente en la docencia a los estudiantes de pregrado. Al margen de estos centros, en los años sesenta-setenta del siglo pasado se construyó una red de hospitales de la Seguridad Social, entre los que se encontraban algunos de alto nivel técnico, pero que no tenían ningún tipo de relación con la universidad. La creación de nuevas universidades y de un número significativo de facultades de medicina hizo que muchas de éstas se pusieran en marcha sin vinculación formal con ningún centro sanitario, de tal manera que, en general, los estudiantes hacían prácticas en los hospitales más próximos, pero acogiéndose siempre a la buena voluntad de los médicos que allí trabajaban o a acuerdos institucionales no demasiado formalizados. Finalmente, en los años ochenta, se fundaron facultades de medicina en algunos grandes hospitales, de tal modo que en ellos se impartía tanto la formación teórica como la práctica de los cursos clínicos.

Esta era la situación en España hace 30 años. Una realidad preocupante por el escaso aprovechamiento de los hospitales públicos para la docencia a los numerosísimos estudiantes de medicina de aquella época, y por la consolidación de dos tradiciones profesionales: la de los médicos vinculados a la universidad, con gran experiencia docente, pero, salvo excepciones, con escasa oportunidad de participar en la organización de los servicios asistenciales; y la de los médicos hospitalarios, con una gran formación clínica, con importante experiencia en la organización y gestión de servicios en los grandes hospitales, pero sin la contrapartida académica o docente.

La respuesta a estos problemas fueron los conciertos. En 1984, los hospitales clínicos se integraron en la red sanitaria pública, dependiendo inicialmente del Insalud y, posteriormente, de las distintas consejerías de sanidad de las comunidades autónomas. Con la intención de armonizar ambas instituciones, universitaria y sanitaria, surgió la necesidad de dictar disposiciones legales y reglamentarias específicas para las facultades de medicina. Estas disposiciones fueron, fundamentalmente, el Real Decreto (RD) de 28 de junio de 1986 [1] y sus posteriores modificaciones, en los que quedaron establecidas las bases generales del régimen de conciertos
Catedrático de Medicina. Decano de la Facultad de Ciencias de la Salud. Hospital Universitario de Gran Canaria Dr. Negrín. Universidad de Las Palmas de Gran Canaria.

E-mail: dec_ccs@ulpgc.es

(c) 2013 FEM 
entre las universidades y las instituciones sanitarias; y la Ley General de Sanidad de 1986, en la que las administraciones educativa y sanitaria se obligaban a garantizar unos adecuados recursos para la docencia de la medicina a todos sus niveles (pre y posgrado, formación de especialistas y formación continuada). En consecuencia, y a partir de ese momento, los mejores centros sanitarios de nuestro país se pudieron utilizar para la enseñanza, en un marco de colaboración cuyos objetivos docentes, asistenciales y de investigación pretendían ser de excelencia.

El planteamiento del RD 1558/86 [1], y de los conciertos que se enmarcan en él, iba en la línea de dar respuesta a los problemas ya comentados. Es evidente que el logro fundamental de todo este proceso fue conseguir los recursos suficientes para la docencia de las profesiones sanitarias, si bien también quedó patente la falta de armonización entre la cultura académica y la estrictamente asistencial. El RD 1558/86 determinó para el hospital universitario una doble dependencia, y para supervisar la buena marcha de los convenios establecidos entre la universidad y las instituciones sanitarias se crearon comisiones de seguimiento de carácter mixto. Si bien las situaciones varían en las diferentes universidades, en general, estas comisiones mixtas han sido inoperantes y el interés de las universidades (y de las administraciones de las que dependen) por la sanidad ha ido decreciendo paulatinamente, lo que ha favorecido una influencia cada vez más relevante de las instituciones sanitarias sobre la enseñanza clínica de los estudiantes de medicina. Además, no se ha conseguido una estructura $u$ operatividad en la participación mutua en los órganos de dirección, tal y como estaba previsto en el RD. Los convenios tampoco se han desarrollado en algunos aspectos relevantes, como la investigación, o presentan deficiencias en otros, como en todo lo relativo a la política de profesorado, en la adecuada equiparación académico-asistencial de los profesores clínicos y en la gestión de las unidades docentes en los hospitales [2].

\section{Práctica clínica y hospital universitario}

Hoy en día, nadie pone en duda la importancia de la formación práctica en la enseñanza de la medicina. La Directiva 2005/36/CE, relativa al reconocimiento de cualificaciones profesionales, establece claramente en su artículo 24.3.d) que la 'formación básica del médico garantizará que el interesado ha adquirido una experiencia clínica adecuada en hospitales bajo oportuna supervisión' [3].
Sin embargo, en la actualidad las prácticas clínicas siguen careciendo, en la mayor parte de los casos, de una clara definición de los objetivos que deben alcanzar en su desarrollo; no están adecuadamente sistematizadas; la participación del estudiante es poco activa y, en general, no existe una evaluación objetiva y fiable de esta docencia práctica, que, habitualmente, se centra en el mero hecho de asistir. Por otra parte, la misma Directiva europea mencionada anteriormente señala, en su artículo 29, que 'los estados miembros condicionarán, [...] el ejercicio de las actividades de médico general en el marco de su régimen general de seguridad social a la posesión de un título de formación..., que en el caso de España es el título de especialista en medicina familiar y comunitaria [3]. Así las cosas, cabría preguntarse si no sería el período de formación especializada, una vez adquirido el bagaje teórico previsto en los planes de estudios, el momento idóneo para la adquisición de la experiencia clínica básica y de las habilidades prácticas necesarias para el ejercicio profesional. De alguna manera, el concepto de troncalidad en la formación de posgrado vendría a reforzar esta idea. En otras palabras, si nuestras facultades no facultan, quizá el planteamiento de las prácticas clínicas y el papel del hospital universitario en la formación de grado podría reformularse.

Finalmente, tampoco se puede olvidar la progresiva diversificación asistencial y la creciente complejidad organizativa del sistema sanitario. El hospital es solamente una parte del proceso asistencial. Existe un nuevo paciente, hoy en día mayoritario, con enfermedades crónicas, para el que el modelo de atención clásico -basado en encuentros cortos, no planificados, para resolver fundamentalmente problemas agudos, con estancias hospitalarias muy breves y tecnificadas y con una importante orientación diagnóstica - ya no da respuesta a sus necesidades. La atención sanitaria actual se realiza en ámbitos cada vez más heterogéneos y predominantemente extrahospitalarios, con un énfasis evidente en las medidas preventivas [4].

\section{Valores del hospital universitario}

El hospital universitario debe ser una institución sanitaria con vocación de excelencia tanto en la generación de conocimiento (investigación), como en su aplicación (asistencia) y su transmisión (docencia). La universidad, además de formar profesionales, tiene la misión trascendental de la creación, el desarrollo, la transmisión y la crítica de la ciencia [4], lo cual es pertinente recordar en un momento 
en el que en España han proliferado de forma insólita numerosas facultades de medicina en estos últimos años. Desde este punto de vista, no se puede concebir una facultad de medicina sin un hospital universitario en el que sean patentes las tres facetas de la gestión del conocimiento médico: asistencia, docencia e investigación.

Pero no basta con recibir estudiantes, con que los alumnos hagan prácticas, para que un hospital sea universitario. Por otra parte, tampoco se precisa un hospital universitario para la docencia de grado. Lo que es necesario es disponer de hospitales generales, centros de salud, consultas de enfermería, unidades de hospitalización domiciliaria, servicios de urgencias extrahospitalarias, centros geriátricos, unidades de cuidados paliativos, laboratorios de simulaciones, etc., para la formación práctica de nuestros estudiantes.

\section{Diez propuestas para mejorar la formación práctica de nuestros estudiantes}

Sin entrar a considerar cuestiones estructurales $u$ organizativas de lo que debería ser un hospital universitario, y sin comentar aspectos políticos o administrativos, como el desproporcionado número de alumnos admitidos en nuestras facultades - que está poniendo en peligro una docencia teórica y práctica de calidad alcanzada en las últimas dos décadas-, a continuación se exponen algunas propuestas o reflexiones respecto al estudiante y su formación práctica clínica:

1. En los tiempos que vivimos, a veces no está de más recordar lo evidente -las verdades de Pero Grullo-, que nuestros alumnos, a veces alentados por determinados profesores, olvidan con facilidad. Estudiar medicina exige muchas horas de estudio. Unos fundamentos teóricos sólidos y rigurosos son imprescindibles para dar sentido a la formación práctica. Una mayor orientación profesional de los estudios universitarios no puede ser la coartada que distraiga del objetivo final: formar médicos capaces de aplicar los conocimientos y el método y el razonamiento científico en el ejercicio de la medicina [5].

2. Es preciso establecer unos objetivos prácticos claros, pertinentes, oportunos, adecuados a la etapa formativa en que se encuentre el estudiante, precisos, realizables y evaluables.

3. No se puede olvidar la existencia de un continuum educativo, no sólo por razones administrativolegales, como se recordaba más arriba, sino por la forma en que se organizan los conocimientos médicos a lo largo de la vida. Esto, en nuestro país, requiere, entre otras cosas, una mayor coordinación entre el Ministerio de Sanidad y el de Educación.

4. Hay que aprovechar lo mejor de todos los entornos educativos, no sólo el hospital universitario, y establecer qué competencias se pueden y deben adquirir en cada uno de ellos. Hay -debe haber- otros hospitales, asociados a la universidad, o centros de salud con funciones más específicas en el entrenamiento del estudiante de grado.

5. Los estudiantes deben ser conscientes de que tendrán que atender, cualquiera que sea su especialidad en el futuro, a un número de pacientes crónicos cada vez mayor. En este sentido, las facultades deben rediseñar el abordaje de la formación práctica de forma que aseguren que el alumno sabe afrontar el increíble reto que supone el cuidado de pacientes a lo largo del tiempo (no sólo cuando está hospitalizado), y cómo las enfermedades crónicas afectan al bienestar de los pacientes y sus familias. También se debe insistir en el papel de los determinantes no biológicos de la enfermedad, porque la medicina necesita realismo en cuanto a la alineación entre la educación médica y las necesidades de la sociedad. No comprender adecuadamente el contexto en el que se va a ejercer la profesión es una fuente importante de insatisfacción. Estas lecciones críticas no pueden ser aprendidas durante las rotaciones tradicionales en el hospital universitario -habitualmente centros de tercer nivel-, en el que los pases de visita se centran en la toma de decisiones rápidas y eficientes, no siempre orientadas a la formación del principiante.

6. Los estudiantes, durante su formación práctica, deben comprender los papeles que juegan otros miembros de los equipos de profesionales sanitarios y cómo afecta el trabajo en equipo a la calidad de la prestación asistencial. Esto es cada vez más trascendente por las evidentes ventajas que supone, especialmente para el cuidado del paciente crónico, la existencia de equipos interdisciplinarios. Las facultades que albergan más de una titulación deberían sacar provecho a esta circunstancia para formar a nuestros estudiantes como futuros miembros de equipos de salud [6].

7. La formación clínica que proporcionan la mayoría de facultades sigue una pauta muy tradicional. Aunque discutible, los estudiantes pueden aprender más con un esquema de prácticas 
clínicas estandarizado y que permita la rotación por un gran número de servicios o unidades asistenciales. Este esquema de prácticas permite a los alumnos hacer unas elecciones acerca de su futuro mucho mejor fundamentadas. Se puede, no obstante, cuestionar la utilidad de este sistema, dado que el estudiante generalmente tiene una participación muy limitada y los beneficios que obtiene se fundamentan, básicamente, en la observación. Sin embargo, al incorporarse a la rutina asistencial de los profesores de prácticas clínicas, al acompañarles en su rutina diaria -lo que en definitiva constituye la filosofía del RD 1558/86-, los estudiantes experimentan lo que constituye el trabajo cotidiano de un médico clínico; observan cómo funcionan los equipos asistenciales; y adquieren una idea mucho más ajustada a la realidad de los retos a los que se enfrentan cada día las diferentes especialidades médicas [7].

8. En cualquier caso, sea cual sea el modelo y el entorno, se debe insistir -como un elemento clave en la formación práctica de los estudiantes- en la utilización del sentido común y en el desarrollo del razonamiento clínico. Muchos estudiantes y residentes sienten una profunda aversión al riesgo y toleran la incertidumbre con dificultad. Se debe hacer hincapié en la importancia que en la práctica clínica tiene pensar, esperar y observar, y en la necesidad de redescubrir el razonamiento clínico como la base fundamental de la práctica profesional. Los profesores deben enseñar a los estudiantes cómo ejercer la medicina basada en las evidencias científicas, y de la forma menos invasiva y más prudente y económica posible [8].

9. Durante las prácticas se aprenden más cosas de las que se enseñan. Los estudiantes adquieren su identidad profesional y las normas de comportamiento no tanto mediante exhortaciones en el aula como mediante la observación de cómo interactúan sus modelos (profesores, tutores, etc.) con los pacientes, con los familiares y con otros profesionales -sanitarios y no sanitarios-, dentro y fuera del hospital. Demasiado a menudo, lo que los estudiantes observan sirve más para alimentar su cinismo que para reforzar los valores del profesionalismo. La disonancia cognitiva entre lo que decimos que creemos y lo que realmente hacemos es enormemente dañina para el médico en formación [9].

10. La investigación forma parte del ejercicio profesional del médico. Es importante transmitir a los alumnos que el médico asistencial debe in- vestigar no sólo por la relevancia de su objetivo más inmediato, sino también para extender la lógica de la investigación científica -basada en el razonamiento deductivo (sacar consecuencias de un principio, proposición o supuesto)- a la práctica clínica, fundamentalmente de carácter inductivo (extraer, a partir de determinadas observaciones o experiencias particulares, el principio general que en ellas está implícito). Esto es un aspecto clave desde un punto de vista formativo tanto en el grado como en el posgrado. El trabajo fin de título de los nuevos grados ofrece una magnífica oportunidad para consolidar esta idea, y el hospital universitario un magnífico entorno para llevarla a cabo.

\section{Conclusión}

Veintiséis años después de que se aprobase la norma que regula los conciertos entre la universidad y las instituciones sanitarias, y tras una década de asunción de las competencias sanitarias por las comunidades autónomas, la estructura, la organización y el funcionamiento de los hospitales universitarios españoles no ha variado significativamente. En las últimas décadas se ha asistido a una difuminación de su papel como centros exclusivos en la docencia práctica de los estudiantes. La reforma que ha propiciado el Espacio Europeo de Educación Superior subraya la importancia de formar a nuestros estudiantes en todos los entornos educativos posibles para asegurar una correcta adquisición de las competencias. En cualquier caso, el hospital universitario continúa teniendo un papel clave en la formación de los estudiantes de medicina, aunque actualmente está sumido en la ambigüedad por dos razones principales: la obsolescencia de la norma que los regula (muy inconcreta y claramente superada por la evolución sanitaria y por el desarrollo legislativo) y la ausencia de una cultura, tanto dentro como fuera de estos hospitales, para verlos como lo que realmente son: centros asistenciales, sí, pero también docentes e investigadores.

Bibliografía

1. Real Decreto $1558 / 1986$, de 28 de junio, por el que se establecen las bases generales del régimen de conciertos entre las universidades y las instituciones sanitarias. BOE de 31 de julio de 1986

2. Cuervas-Mons Martínez V. Facultad y hospital: una estructura bipolar y específica para los estudios de medicina. Educ Med 2009; 12 (Supl 3): S13-4.

3. Directiva 2005/36/CE del Parlamento Europeo y del Consejo, 
de 7 de septiembre de 2005, relativa al reconocimiento de cualificaciones profesionales.

4. Civeira Murillo F. El hospital como centro nuclear para mejorar la enseñanza de la Medicina. Educ Med 2010; 13 (Supl 1): S33-5.

5. Álvarez-Sala Walther JL, Nieto Barbero MA, Rodríguez Trigo G. La formación clínica como piredra angular de los estudios de Medicina. Educ Med 2010; 13 (Supl 1): S25-31.

6. Frenk J, Chen L, Bhutta ZA, Cohen J, Crisp N, Evans T, et al.
Health professionals for a new century: transforming education to strengthen health systems in an interdependent world. Lancet 2010; 376: 1923-58.

7. Xu R. A differentiation diagnosis -specialization and the medical student. N Engl J Med 2011; 365: 391-3.

8. Palfrey S. Daring to practice low-cost mediciine in a high-tech era. N Engl J Med 2011; 364: e21.

9. Rodríguez de Castro F. Proceso de Bolonia (V): el currículo oculto. Educ Med 2012; 15: 13-22. 


\section{El hospital como centro de enseñanza de la medicina}

Jesús Millán Núñez-Cortés

La enseñanza de la medicina se encuentra obligatoriamente ligada a la enseñanza en los hospitales. Por tanto, frente a los desafíos sanitarios del futuro, es una obligación y una exigencia para la sociedad reforzar la triple misión asistencial, docente e investigadora de los hospitales universitarios como centros de excelencia profesional en estos tres aspectos.

El desarrollo de tales funciones se va a desarrollar cada vez en un ambiente de mayor exigencia, en el que el carácter ejemplar del hospital universitario puede ser determinante a la hora de aplicar las mayores y mejores evidencias científicas, la introducción de nuevas tecnologías o la implementación de las innovaciones en el terreno de la educación médica.

Por tanto, parece pertinente actualizar el papel que el hospital universitario tiene en el seno del sistema sanitario, su función en el -siempre cambiante- sistema sanitario, y -eventualmente- considerar la posible reubicación para dicha función, señalando los principios generales que primen la coordinación en el cumplimiento de sus misiones en un escenario de calidad.

El modelo clásico de hospital, hasta ahora el más utilizado en la enseñanza de la medicina, no siempre satisface las demandas sanitarias de la sociedad, ni tampoco un sistema de investigación competitiva o de enseñanza avanzada. Este último aspecto puede ser tanto más notable si en las instituciones hospitalarias no se percibe con notoriedad la vocación docente de los profesionales, y de la propia institución en su conjunto, que ha de incorporar la cultura de formación a todos sus niveles. Parece, por tanto, que resulta obligada una especialización en la docencia como uno de los rasgos distintivos de un hospital universitario.

Para llevar a cabo una correcta enseñanza/aprendizaje de la medicina, es necesaria la incorporación activa del alumno a un lugar concreto e integrado en un grupo concreto. Es necesario un entorno específico con una metodología específica para que se encuentren el maestro y el alumno con el enfermo. Y este modelo de interacción ha de realizarse en un hospital en el que el carácter universitario no sea sólo un adjetivo, sino una cualidad.

Los requerimientos definidos para llevar a cabo en un hospital labores 'universitarias' (docentes y de investigación) resultan bastante imprecisos, y eso no se percibe cuando se definen los requerimientos asistenciales, que son claros y netos. Ocurre igual cuando se analizan los objetivos del hospital universitario, que permiten identificar claramente cuáles son los asistenciales, cosa que no ocurre con los objetivos docentes o de investigación. Finalmente, la gestión del hospital universitario está centrada en la asistencia, pero no en la docencia o en la investigación, que quedan en un 'segundo orden' mucho menos regulado y, por ende, sin un protagonismo claro. La docencia ni se tiene en cuenta en la estructura (espacios físicos, órganos de gobierno), ni en los procesos desarrollados (organización), ni en los resultados (indicadores, incentivos).

En consecuencia, la docencia universitaria en los hospitales universitarios se percibe (y resulta ser), con frecuencia, como una función marginal, porque el modelo organizativo no la incorpora al mismo nivel que la asistencial ni el presupuesto hospitalario está repercutido por la docencia universitaria; en otras palabras, la 'cultura' y el funcionamiento diario del hospital no están impregnados por la función docente propia del hospital universitario. Por tanto, a veces, la función del profesorado no está bien definida, y ello se debe a una insuficiente definición de las relaciones funcionales y económicas, que pueden no ser ni siquiera uniformes (por normas autonómicas distintas), lo que está agravado por una cierta dificultad de interlocución (no siempre se utiliza 'el mismo lenguaje').
Catedrático de Medicina de la Universidad Complutense de Madrid. Director de la Cátedra de Educación Médica Fundación Lilly-UCM

E-mail:

jesus.millan.nunezcortes@ madrid.org

(C) 2013 FEM 
Por lo tanto, es erróneo pensar que poseer infraestructura, cumplir algunas necesidades y haber sido concertado pueda ser suficiente para que un hospital se considere como universitario, cuando -en realidad- eso sólo no garantiza que la docencia que allí se realice alcance la excelencia que se pretende, sin que se definan previamente numerosas cuestiones que la realidad y la experiencia han ido señalando: presencia de un número mínimo o crítico de profesores funcionarios en el hospital (los 'profesionales' de la docencia), garantía de unos espacios mínimos para realizar la docencia en condiciones adecuadas, salvaguarda de la dedicación docente del médico, establecimiento de objetivos docentes y de investigación como incentivación para el personal hacia tales funciones, consideración de una financiación ad hoc, etc.

Estas carencias son tanto más acusadas cuando consideramos la enseñanza de grado (hasta ahora denominada de 'pregrado'). En efecto, la enseñanza de posgrado, y sobre todo la formación de especialistas, se encuentra fuertemente regulada: las estructuras de apoyo, los espacios necesarios, la organización del personal docente (jefe de estudios, tutores, unidades docentes), el cumplimiento de los programas, las plazas docentes disponibles, etc. Casi todo lo relativo a la formación de especialistas está predefinido y, por tanto, se puede auditar (y de hecho se audita para corregir las desviaciones o las carencias). Como ya se ha señalado, para la enseñanza de grado todos estos prerrequisitos no se han definido más que de una forma genérica $y$, por lo tanto, no se audita su cumplimiento. Podría suceder que algunos hospitales universitarios no cumplieran sus obligaciones como tales, o que no dispusieran de una infraestructura docente adecuada, y al no ser auditados como tales no dejarían de ser 'universitarios' (como sería lógico en ese caso).

\section{Misión, visión y valores del hospital universitario}

Se precisa enfatizar en el papel trascendental que tiene el hospital universitario para la enseñanza de las profesiones sanitarias, y muy especialmente para la enseñanza de la medicina, proceso en el que todas las recomendaciones emanadas de la educación médica aconsejan nuclear alrededor de la institución hospitalaria.

\section{Misión}

El hospital universitario ha de ser una institución sa- nitaria con vocación académica explícitamente reflejada en sus estructura, en sus procesos y en el análisis de sus resultados, organizada para dar respuesta a las necesidades sociales a través de una visión unitaria de la asistencia, docencia e investigación, y en un entorno que garantice la consecución de los objetivos en cada una de estas tres funciones.

\section{Visión}

El hospital universitario es una institución orientada a mejorar la salud de la población, y cuya cultura académica le permite y le obliga a realizar una asistencia sanitaria de excelencia, una investigación competitiva y de calidad, y una formación completa a profesionales y futuros profesionales, cumpliendo los estándares de calidad en cada uno de sus niveles.

\section{Valores}

- El hospital universitario ha de ser una institución sanitaria:

- Centrada en el ciudadano enfermo y en el alumno.

- Orientada a crear un entorno que garantice la satisfacción de los profesionales que realizan asistencia, docencia e investigación.

- Prestando asistencia sanitaria de excelencia.

- Ofreciendo una enseñanza de la medicina y de las profesiones sanitarias a todos sus niveles (grado, posgrado, especialistas y formación continuada).

- Liderando activamente la investigación biomédica.

- Con una vocación firme como motor y gestor del conocimiento científico a través de su adquisición (investigación), de su transmisión (docencia), y de su aplicación (asistencia).

- Con capacidad para adaptar su estructura y sus procesos a la consecución de unos mejores resultados en los tres campos de actuación: asistencia, docencia e investigación.

En consecuencia, y después del análisis de la situación actual y de cara a considerar el hospital auténticamente como un centro de enseñanza de la medicina, se impone:

1. Actualizar y generar normas generales que regulen la estructura y el funcionamiento de los hospitales universitarios.

2. Definir e incorporar estándares focalizados no sólo en la asistencia, sino también en la docencia e investigación, para su correcto cumplimiento y evaluación. 
3. Incorporar al hospital universitario las funciones, la organización y la cultura propias de una organización que contemple la docencia y la investigación como uno de sus rasgos distintivos.

4. Facilitar que el hospital universitario se transforme en una institución en la que se gestione el conocimiento en todas sus facetas: adquisición (in- vestigación), aplicación (asistencia) y transmisión (docencia).

5. Optimizar la utilización de los recursos asistenciales para el cumplimiento de los principios que se incorporan a los nuevos planes de estudio con el fin de garantizar las mejores prácticas en educación médica. 


\section{Relaciones universidad-sistema sanitario}

José Javier Castrodeza

- Principio básico. La estructura asistencial del sistema sanitario, tanto en sus recursos materiales como humanos, debe poder utilizarse para la docencia pregraduada, posgraduada y continuada de los profesionales sanitarios, del mismo modo que el sistema universitario debe servir para su reciclaje (RD 1558/86).

- Marco Normativo. Ley Orgánica 11/1983, de Reforma Universitaria. Ley 14/1986, General de Sanidad. Real Decreto 1558/1986. Ley Orgánica 6/2001, de Universidades. Ley 44/2003, de Ordenación de Profesiones Sanitarias. Marco legislativo autonómico.

\section{- Finalidad.}

- Formación de pregrado y máster.

- Formación de doctorado.

- Formación continuada y títulos propios (títulos de especialista universitario).

- Formación especializada en sus diversas opciones y abordajes.

\section{EJERCICIO PROFESIONAL}

\section{Conciertos universidad-sistema sanitario}

- Objetivos: docentes, asistenciales e investigadores.

- Profesiones: medicina, farmacia, enfermería, fisioterapia, otras.

- Órganos de coordinación: comisiones mixtas.

- Reconocimiento: máximo nivel institucional universitario y sanitario.

- Personal docente: profesor vinculado, profesor asociado, otros.

- Reconocimiento institucional. Hospital y centro universitario.

- Colaboración. Recursos humanos, tecnológicos y materiales.

\section{Objetivos docentes}

- Promover la máxima utilización de los recursos sanitarios hospitalarios y extrahospitalarios, humanos y materiales para la docencia.

- Cooperar en el mantenimiento de la cualificación de los profesionales de la salud a su más alto nivel, cuidando su actualización y reciclaje, y favoreciendo su incorporación a la docencia universitaria.

\section{Objetivos asistenciales}

- Cooperar para que las investigaciones y enseñanzas universitarias en medicina, enfermería, farmacia y demás profesiones sanitarias puedan ser utilizadas para la mejora constante de la atención sanitaria.

- Prever que coincidan la mayor calidad asistencial con la consideración de hospital universitario o asociado a la universidad.

\section{Objetivos investigadores}

- Potenciar la investigación en las ciencias de la salud, coordinando las actividades de las universidades con las de las instituciones sanitarias, para una mejor utilización de los recursos humanos y materiales.

- Favorecer el desarrollo de los departamentos universitarios en las áreas de la salud, potenciando su coordinación con las unidades de investigación de los hospitales y estimulando las vocaciones investigadoras.

\section{Comisión mixta}

Es una comisión paritaria con funciones específicas, sin perjuicio de las competencias propias de cada una de las instituciones:

- Velar por la correcta aplicación del concierto.
Director general de Ordenación Profesional. Ministerio de Sanidad, Servicios Sociales e Igualdad.

E-mail:

sdgcai@msssi.es

(C) 2013 FEM 
Figura 1. Tendencia plazas titulación de medicina.

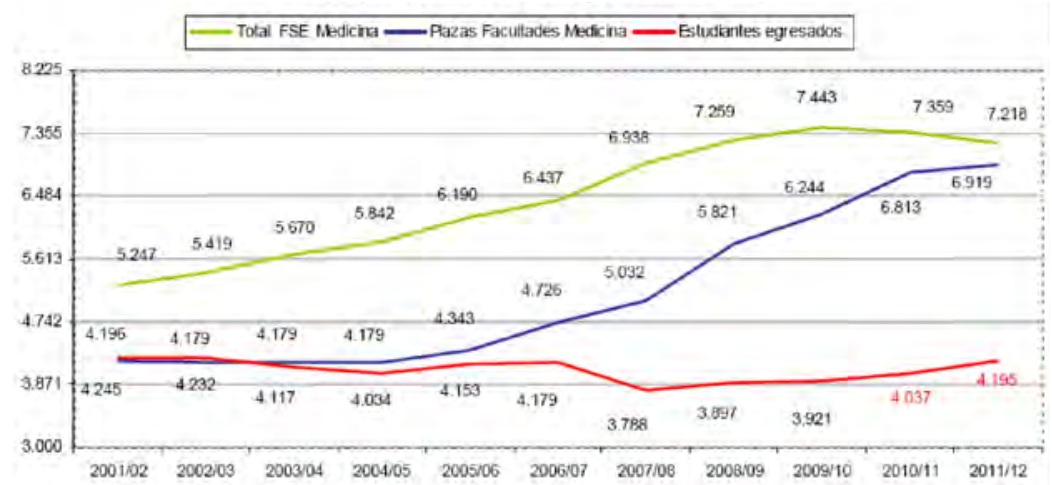

- Proponer la reducción, ampliación o transformación del número de plazas vinculadas y de profesores asociados.

- Establecer el plan de coordinación de la realización de las prácticas.

- Proponer las fórmulas de coordinación entre las actividades docentes.

- Proponer las fórmulas de participación con el fin de garantizar la coordinación de la estructura departamental prevista en la Ley Orgánica de Reforma Universitaria con la estructura funcional de la institución sanitaria.

- Proponer fórmulas que permitan la valoración de la condición de profesor con plaza vinculada en la convocatoria para la provisión de puestos de jefaturas.

\section{Personal docente}

- Los profesores que ocupen una plaza vinculada desarrollarán el conjunto de sus funciones docentes, investigadoras y asistenciales en una misma jornada.

- La dedicación horaria semanal de dicha jornada está establecida.

- La comisión mixta universidad-institución sanitaria establecerá las fórmulas de coordinación.

- La plaza vinculada podrá ser a tiempo completo y a tiempo parcial. Se requerirá el reconocimiento.

- La duración de la jornada laboral será la legalmente establecida para el personal con plaza exclusivamente asistencial en centros sanitarios públicos.

- Todas las retribuciones del personal que ocupe plaza vinculada se abonarán en una única nómina por la universidad.
Figura 2. Tendencia adjudicatarios MIR por género.

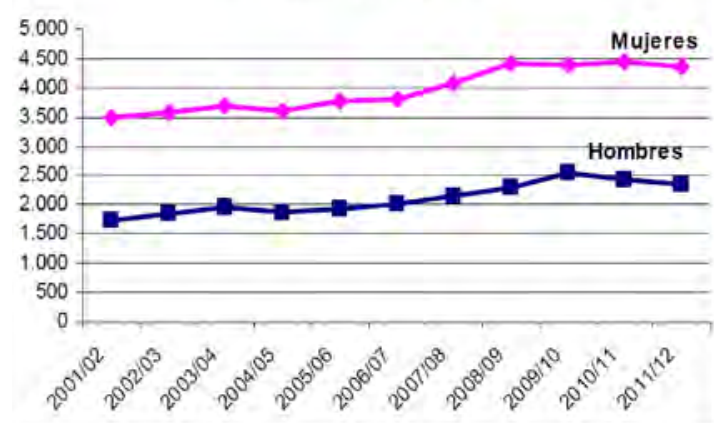

- Los profesores que desempeñen plaza vinculada tendrán los derechos y deberes inherentes a su condición de cuerpos docentes de universidad y de personal estatutario del régimen correspondiente de la Seguridad Social o de la institución concertada que corresponda cuando ésta no pertenezca a aquélla.

\section{Valor aportado por los convenios/conciertos.}

\section{RD 1558/1986}

- En los años noventa se desarrollan estos acuerdos.

- Mayor nivel de desarrollo para los estudios de medicina.

- Actualizaciones en las comunidades autónomas por asumir competencias.

- Cierta variabilidad y especificidad en función de la comunidad autónoma.

- Extensión a otras titulaciones de ciencias de la salud.

- Escenario facilitador de una mejor investigación básica/clínica.

- Mayor implicación de los profesionales del sistema en la docencia.

- Optimización de recursos investigadores, formativos y estructurales.

- Este escenario integrado ha influido en la calidad del Sistema Nacional de Salud.

\section{Nuevos escenarios que se deben considerar en los futuros convenios/conciertos}

Algunas reflexiones:

- Han transcurrido casi 30 años del modelo jurídico regulador de las relaciones. 
- Las comunidades autónomas han desarrollado nuevos marcos de colaboración entre sus instituciones.

- Se han modificado los planes de estudio (Bolonia).

- El peso de la investigación en el desarrollo profesional del 'vinculado' es condicionante.

- Hay menos interés por el entorno docente universitario.

- El sistema sanitario posee experiencia y gran potencial formativo.

- La investigación tiene cada vez más un marcado carácter 'aplicativo'.

- La gestión del conocimiento ha de ser más eficiente y con valor de retorno.

- El mundo sanitario ocupa un papel relevante como generador de riqueza.

- La sociedad del bienestar y el conocimiento de sus profesionales están relacionados.

\section{Profesiones sanitarias}

Ley 44/2003, de Ordenación de Profesiones Sanitarias:

- Determina las profesiones sanitarias.

- Determina la estructura y formación en especialidades en ciencias de la salud.

- Programas de formación.

- Catálogos de especialidades y áreas de capacitación.
- Diplomas de acreditación y diplomas de acreditación avanzada.

- Ejercicio privado de las profesiones sanitarias.

\section{Algunos datos sobre \\ determinadas profesiones sanitarias}

\begin{tabular}{lcc} 
Convocatoria 2011/12 & Plazas convocadas & Aspirantes admitidos \\
\hline Medicina & 6.707 & 13.544 \\
\hline Enfermería & 1.002 & 17.828 \\
\hline Farmacia & 309 & 1.388 \\
\hline Psicología & 171 & 3.693 \\
\hline
\end{tabular}

\section{Conclusión}

La relación universidades-sistema sanitario es determinante en la sociedad del bienestar, debido a la importancia que desde todos sus campos tiene el sector salud. El respeto y reconocimiento que cada una de estas instituciones tiene no impide avanzar en una relación sólida, coordinada e integrada en beneficio de nuestro sistema educativo y nuestro Sistema Nacional de Salud, en beneficio de la sociedad. 